C. Tezer

Nagoya Math. J.

Vol. 134 (1994), 29-55

\title{
AUTOMORPHISM GROUPS OF A CLASS OF EXPANDING ATTRACTORS ${ }^{1}$
}

\author{
CEM TEZER
}

\section{Introduction and statement of results}

In the following, for any group $G$ and any $g \in G, \operatorname{Ad}[g]$ stands for the inner automorphism defined by $\operatorname{Ad}[g](x)=g x g^{-1}$ for any $x \in G$.

Given a group endomorphism $\alpha: G \rightarrow G$ let $\mathscr{G}^{\sim}(\alpha)$ be the set of triples $(\varphi, m, g)$ where $g \in G, m \in Z$ and $\varphi$ is an endomorphism of $G$ effecting a shift equivalence ([11], [14], [15]) of $\alpha$ to $\operatorname{Ad}[g] \circ \alpha$. On $\mathscr{G}^{\sim}(\alpha)$ we consider a binary relation “ " defined by

$$
(\varphi, m, g) \sim\left(\varphi^{\prime}, m^{\prime}, g^{\prime}\right)
$$

iff there exist $x \in G, T \in Z$ such that

$$
\begin{gathered}
T \geq m, m^{\prime} \\
x g=g^{\prime} \alpha(x) \\
\varphi^{\prime} \circ \alpha^{T-m^{\prime}}=\operatorname{Ad}[x] \circ \varphi^{\circ} \alpha^{T-m},
\end{gathered}
$$

and a binary operation

$$
\mu: \mathscr{G}^{\sim}(\alpha) \times \mathscr{G}^{\sim}(\alpha) \rightarrow \mathscr{G}^{\sim}(\alpha)
$$

defined by

$$
\mu((\varphi, m, g),(\phi, n, h))=\left(\varphi^{\circ} \phi, m+n, \varphi(h) g\right) .
$$

The opening passages of the next section are devoted to the fairly routine yet technically indispensable proofs of the following facts:

Received June 24, 1991.

1 This work has been supported by the Turkish Council of Scientific and Technological Research. 
1.1. Lemma. “ " is an equivalence relation.

1.2. Lemma. $\mu: \mathscr{G}^{\sim}(\alpha) \times \mathscr{G}^{\sim}(\alpha) \rightarrow \mathscr{G}^{\sim}(\alpha)$ is a well-defined map.

To be precise: If $\varphi, \phi$ are endomorphisms of $G$ effecting shift equivalences of $\alpha$ to $\operatorname{Ad}[g] \circ \alpha, \operatorname{Ad}[h] \circ \alpha$ respectively, then $\varphi^{\circ} \phi$ effects a shift equivalence of $\alpha$ to $\operatorname{Ad}[\varphi(h) g] \circ \alpha$.

1.3. Lemma. $\mu$ is stable with respect to “ $\sim$ ".

In other words, if

$$
(\varphi, m, g) \sim\left(\varphi^{\prime}, m^{\prime}, g^{\prime}\right)
$$

and

$$
(\phi, n, h) \sim\left(\phi^{\prime}, n^{\prime}, h^{\prime}\right)
$$

then

$$
\left(\varphi^{\circ} \phi, m+n, \varphi(h) g\right) \sim\left(\varphi^{\prime} \circ \psi^{\prime}, m^{\prime}+n^{\prime}, \varphi^{\prime}\left(h^{\prime}\right) g^{\prime}\right) .
$$

Having established these results, we are in a position to introduce yet another natural object: Let's denote the set of $\sim$-equivalence classes in $\mathscr{G}^{\sim}(\alpha)$ by $\mathscr{G}(\alpha)$. In view of $1.1,1.2,1.3$, it is seen that the $\operatorname{set} \mathscr{G}(\alpha)$ has a well-defined binary operation. We round off this tedious list of technical observations by

1.4. Lemma. $\mathscr{G}(\alpha)$ is a group.

Let $a: X \rightarrow X$ be any continuous map of a compact space into itself. We consider the simple inverse limit of the form

$$
\Sigma_{a}=\lim _{\leftarrow}(X, a)=\left\{\left(x_{\imath}\right)_{i \in Z^{+}} \in X^{Z^{+}}, a\left(x_{i+1}\right)=x_{i}, i \in Z^{+}\right\}
$$

and the map

$$
\sigma_{a}: \Sigma_{a} \rightarrow \Sigma_{a}
$$

called the "shift", defined by

$$
\sigma_{a}\left(\left(x_{i}\right)_{i \in Z^{+}}\right)=\left(x_{i+1}\right)_{i \in Z^{+}} .
$$

$\sigma_{a}$ is clearly a homeomorphism. We shall be mainly interested in the case where $a$ is an expanding map of a compact smooth manifold ([9], [6]). In this case, $\sigma_{a}$ is 
known to be an Axiom-A attractor, a so called "expanding attractor" ([10], [15], [11]).

Denoting for any continuous map $b: Y \rightarrow Y$, the group of homeomorphisms of $Y$ commuting with $b$ by Aut $(b)$ we can formulate the main result of the present work as follows:

1.5. Proposition. If $a: X \rightarrow X$ is an expanding map of a compact smooth manifold $X$ into itself with a fixed point $x_{*} \in X$, then

$$
\operatorname{Aut}\left(\sigma_{a}\right) \simeq \mathscr{G}\left(a_{\#}\right)
$$

where

$$
a_{\#}: \pi_{1}\left(X, x_{*}\right) \rightarrow \pi_{1}\left(X, x_{*}\right)
$$

is the endomorphism of the fundamental group induced by $a$.

This proposition is, in fact, just another tribute to a fundamental result (Theorem 4 (I) in [9]) due to M. Shub and J. Tate which indicates that an expanding map has a very rigid geometry, entirely determined by algebra at the level of fundamental groups.

Although its definition is complicated, it is possible to compute $\mathscr{G}(\alpha)$ in some concrete cases. We have considered the endomorphisms

a) $\varphi(p): Z \rightarrow Z$ (the additive group) with $p \in Z,|p| \neq 0,1$ defined by $\varphi(p)(x)=p x$ for all $x \in Z$.

b) $\varphi(p, q, r): G=\left\langle A, B \mid A B=B A^{-1}\right\rangle \rightarrow G \quad$ with $\quad p, q, r \in Z,|p| \neq$ 0,1 and $r$ odd, $|r| \neq 1$, defined by

$$
\varphi(p, q, r)\left\{\begin{array}{l}
A \rightarrow A^{p} \\
B \rightarrow A^{q} B^{r} .
\end{array}\right.
$$

These endomorphisms arise in connection with the expanding maps $a(p)$ and $a(p, q, r)$ of the circle and the klein bottle respectively; indeed

$$
\begin{aligned}
(a(p))_{\#} & =\varphi(p) \\
(a(p, q, r))_{\#} & =\varphi(p, q, r)
\end{aligned}
$$

([11], [12]).

If $r$ is any integer, $|r| \geq 2$, let $P R(r)$ denote the set of prime factors of $r$ and let 


$$
r=(-1)^{\tau} r_{1}^{k_{1}} r_{2}^{k_{2}} \cdots r_{s}^{k_{s}}
$$

be the unique factorisation of $r$ into its-positive-prime factors, $s=\# P R(r)$. There is an action

$$
\alpha(r): Z_{2} \oplus Z^{P R(r)} \rightarrow \operatorname{Aut}\left(Z_{r-1}\right)
$$

defined by

$$
\begin{gathered}
\alpha(r)\left(\left(\rho,\left(\zeta_{1}, \zeta_{2}, \ldots, \zeta_{s}\right)\right)\right)(x) \\
=\left[(-1)^{\rho+\tau} \prod_{i=1}^{s} r_{i}^{\zeta_{i}+T k_{i}}\right] x
\end{gathered}
$$

where $T$ is any integer with $\zeta_{i}+T k_{i} \geq 0$ for all $i=1,2, \ldots$, s. It can be easily checked that $\alpha(r)$ is a well-defined action. Now we are in a position to state our computational results. Let $\square_{\alpha}$ denote the semi-direct product of groups via the action $\alpha$.

1.6. Proposition.

$$
\operatorname{Aut}\left(\sigma_{\alpha(p)}\right) \simeq \mathscr{G}(\varphi(p)) \simeq\left(Z_{2} \oplus Z^{P R(p)}\right) \square_{\alpha(p)} Z_{p-1} .
$$

\subsection{Proposition.}

$$
\begin{aligned}
\operatorname{Aut}\left(\sigma_{\alpha(p, q, r)}\right) \simeq & \mathscr{G}(\varphi(p, q, r)) \simeq \\
& \begin{cases}Z^{P R(p)} \oplus\left[\left(Z_{2} \oplus Z^{P R(r)}\right) \square_{\alpha(r)} Z_{r-1}\right] & \text { if } p \equiv 0(\bmod 2) \\
\left(Z_{2} \oplus Z^{P R(p)}\right) \oplus\left[\left(Z_{2} \oplus Z^{P R(r)}\right) \square_{\alpha(r)} Z_{r-1}\right] & \text { if } p \equiv 1(\bmod 2) .\end{cases}
\end{aligned}
$$

It should be noted, in the case of expanding maps of the circle, $a(p)$ is completely determined by $\operatorname{Aut}\left(\sigma_{a(p)}\right)$. This is to be contrasted with the klein bottle situation where $\operatorname{Aut}\left(\sigma_{a(p, q, r)}\right)$ determines solely the number of prime factors of $p$ and misses $q$ entirely. One might tentatively conjecture that, within the framework of expanding attractors, Aut $\left(\sigma_{a}\right)$ carries good information about or completely determines $a$ only when $\sigma_{a}$ is a homogeneous system ([13]).

In our opinion, there are two aspects of the main result 1.5 which are noteworthy: The first and the more immediately discernible is its being the inevitable step in any computational venture as regards automorphism groups of expanding attractors. The second and possibly the less obvious is that $\operatorname{Aut}\left(\sigma_{a}\right)$ has a group structure $\mathscr{G}(\alpha)$ which has a completely independent existence of its own in a purely group theoretical setting. Thus it is to be expected that many concepts which 
naturally arise in connection with dynamical systems and their automorphism groups, such as periodic points and gyration numbers can be incorporated into pure algebra ([1], [2])

Finally we notice that the automorphism groups of expanding attractors appear to differ sharply from those of subshifts of the finite type. Expanding attractors have fair sized automorphism groups whereas in the case of subshifts of the finite type the automorphism groups are always large enough to contain the direct product of any countable family of finite groups ([4], [1]). In the automorphism group of a subshift of the finite type the centre consists of the infinite cyclic group generated by the shift itself ([8], [4], [1]). In contrast, the automorphism group of an expanding attractor seems to contain large, highly commutative pieces.

\section{Proofs of $1.1,1.2,1.3,1.4$}

2.1. Proof of 1.1. Reflexivity of " $\sim$ " is obvious. Replacing $x$ by $x^{-1}$ in the defining equations, it can be seen that " $\sim$ " is symmetric. As for transitivity, let's consider

$$
(\varphi, m, g),\left(\varphi^{\prime}, m^{\prime}, g^{\prime}\right),\left(\varphi^{\prime \prime}, m^{\prime \prime}, g^{\prime \prime}\right) \in \mathscr{G}^{\sim}(\alpha)
$$

with

$$
\begin{gathered}
(\varphi, m, g) \sim\left(\varphi^{\prime}, m^{\prime}, g^{\prime}\right) \\
\left(\varphi^{\prime}, m^{\prime}, g^{\prime}\right) \sim\left(\varphi^{\prime \prime}, m^{\prime \prime}, g^{\prime \prime}\right) .
\end{gathered}
$$

Therefore, there exist $T, T^{\prime} \in Z, x, x^{\prime} \in G$ such that

$$
\begin{aligned}
T & \geq m, m^{\prime} \\
x g & =g^{\prime} \alpha(x) \\
\varphi^{\prime} \circ \alpha^{T-m^{\prime}} & =\operatorname{Ad}[x] \circ \varphi^{\circ} \alpha^{T-m}
\end{aligned}
$$

and

$$
\begin{aligned}
T^{\prime} & \geq m^{\prime}, m^{\prime \prime} \\
x^{\prime} g^{\prime} & =g^{\prime \prime} \alpha\left(x^{\prime}\right) \\
\varphi^{\prime \prime} \circ \alpha^{T-m^{\prime \prime}} & =\operatorname{Ad}\left[x^{\prime}\right] \circ \varphi^{\prime} \circ \alpha^{T-m^{\prime}} .
\end{aligned}
$$

Let $S=\max \left(T, T^{\prime}\right)$ and $y=x^{\prime} x$. We have

$$
S \geq m, m^{\prime \prime}
$$


and

$$
y g=x^{\prime} x g=x^{\prime} g^{\prime} \alpha(x)=g^{\prime \prime} \alpha\left(x^{\prime}\right) \alpha(x)=g^{\prime \prime} \alpha\left(x^{\prime} x\right)=g^{\prime \prime} \alpha(y)
$$

and finally

$$
\begin{aligned}
\varphi^{\prime \prime} \circ \alpha^{S-m^{\prime \prime}}=\operatorname{Ad}\left[x^{\prime}\right] \circ \varphi^{\prime} \circ \alpha^{S-m^{\prime}}=\operatorname{Ad}\left[x^{\prime}\right] \circ \operatorname{Ad}[x] \circ \varphi^{\circ} \alpha^{S-m} \\
=\operatorname{Ad}\left[x^{\prime} x\right] \circ \varphi^{\circ} \alpha^{S-m} \\
=\operatorname{Ad}[y] \circ \varphi^{\circ} \alpha^{S-m}
\end{aligned}
$$

which shows that

$$
(\varphi, m, g) \sim\left(\varphi^{\prime \prime}, m^{\prime \prime}, g^{\prime \prime}\right)
$$

In the following we shall make repeated use of the notation $g_{m}$ standing for the element of $G$, defined inductively for each $g \in G, m \in Z^{+}$by

$$
g_{0}=e
$$

and

$$
g_{k+1}=g \alpha\left(g_{k}\right)
$$

(equivalently $g_{k+1}=g_{k} \alpha^{k}(g)$ ). A moment's reflection shows that $g_{m}$ is concocted so as to satisfy

$$
(\operatorname{Ad}[g] \circ \alpha)^{m}=\operatorname{Ad}\left[g_{m}\right] \circ \alpha^{m}
$$

for each $m \in Z^{+}$.

2.2. Proof of 1.2. Given $(\varphi, m, g) \in \mathscr{G}^{\sim}(\alpha),(\phi, n, h) \in \mathscr{G}^{\sim}(\alpha)$, there exist $\varphi^{\prime}: G \rightarrow G, \phi^{\prime}: G \rightarrow G, M, N \in Z^{+}$such that

$$
\begin{gathered}
\varphi^{\circ} \alpha=(\operatorname{Ad}[g] \circ \alpha) \circ \varphi \\
\varphi^{\prime} \circ(\operatorname{Ad}[g] \circ \alpha)=\alpha^{\circ} \varphi^{\prime} \\
\varphi^{\prime} \circ \varphi=\alpha^{M} \\
\varphi^{\circ} \varphi^{\prime}=(\operatorname{Ad}[g] \circ \alpha)^{M}=\operatorname{Ad}\left[g_{M}\right] \circ \alpha^{M}
\end{gathered}
$$

and

$$
\begin{gathered}
\phi^{\circ} \alpha=(\operatorname{Ad}[h] \circ \alpha) \circ \phi \\
\phi^{\prime}(\operatorname{Ad}[h] \circ \alpha)=\alpha \circ \psi^{\prime} \\
\phi^{\prime} \circ \phi=\alpha^{N} \\
\phi^{\circ} \psi^{\prime}=(\operatorname{Ad}[h] \circ \alpha)^{N}=\operatorname{Ad}\left[h_{N}\right] \circ \alpha^{N} .
\end{gathered}
$$


Now writing $\varphi^{\prime \prime}=\operatorname{Ad}\left[h_{M}\right] \circ \varphi^{\prime}$ we observe that

$$
\begin{aligned}
\varphi \circ(\operatorname{Ad}[h] \circ \alpha) & =\operatorname{Ad}[\varphi(h)] \circ \varphi \circ \alpha \\
& =\operatorname{Ad}[\varphi(h)] \circ \operatorname{Ad}[g] \circ \alpha \circ \varphi \\
& =(\operatorname{Ad}[\varphi(h) g] \circ \varphi
\end{aligned}
$$

and

$$
\begin{aligned}
\varphi^{\prime \prime} \circ(\operatorname{Ad}[\varphi(h) g] \circ \alpha) & =\operatorname{Ad}\left[h_{M}\right] \circ \varphi^{\prime} \circ \operatorname{Ad}[\varphi(h) g] \circ \alpha \\
& =\operatorname{Ad}\left[h_{M}\right] \circ \operatorname{Ad}\left[\varphi^{\prime} \circ \varphi(h)\right] \circ \varphi^{\prime} \circ \operatorname{Ad}[g] \circ \alpha \\
& =\operatorname{Ad}\left[h_{M}\right] \circ \operatorname{Ad}\left[\alpha^{M}(h)\right] \circ \alpha^{\circ} \varphi^{\prime} \\
& =\operatorname{Ad}\left[h_{M} \alpha^{M}(h)\right] \circ \alpha^{\circ} \varphi^{\prime} \\
& =\operatorname{Ad}\left[h \alpha\left(h_{M}\right)\right] \circ \alpha^{\circ} \varphi^{\prime} \\
& =(\operatorname{Ad}[h] \circ \alpha) \circ\left(\operatorname{Ad}\left[h_{M}\right] \circ \varphi^{\prime}\right) \\
& =(\operatorname{Ad}[h] \circ \alpha) \circ \varphi^{\prime \prime}
\end{aligned}
$$

and

$$
\varphi^{\prime \prime} \circ \varphi=\operatorname{Ad}\left[h_{M}\right] \circ \varphi^{\prime} \circ \varphi=\operatorname{Ad}\left[h_{M}\right] \circ \alpha^{M}=(\operatorname{Ad}[h] \circ \alpha)^{M} .
$$

Finally

$$
\begin{aligned}
\varphi^{\circ} \varphi^{\prime \prime} & =\varphi^{\circ} \operatorname{Ad}\left[h_{M}\right] \circ \varphi^{\prime} \\
& =\operatorname{Ad}\left[\varphi\left(h_{M}\right)\right] \circ \varphi^{\circ} \varphi^{\prime} \\
& =\operatorname{Ad}\left[\varphi\left(h_{M}\right)\right] \circ(\operatorname{Ad}[g] \circ \alpha)^{M} \\
& =\operatorname{Ad}\left[\varphi\left(h_{M}\right) g_{M}\right] \circ \alpha^{M}
\end{aligned}
$$

which gives upon writing $h_{M}=h_{M-1} \alpha^{M-1}(h), g_{M}=g_{M-1} \alpha^{M-1} \alpha(g)$

$$
\begin{gathered}
\varphi^{\circ} \varphi^{\prime \prime}=\operatorname{Ad}\left[\varphi\left(h_{M-1}\right) \varphi\left(\alpha^{M-1}(h)\right) g_{M-1} \alpha^{M-1}(g)\right] \circ \alpha^{M} \\
=\operatorname{Ad}\left[\varphi\left(h_{M-1}\right)\right] \circ \operatorname{Ad}\left[g_{M-1}\left(\operatorname{Ad}\left[g_{M-1}^{-1}\right] \circ \varphi^{\circ} \alpha^{M-1}(h)\right)\right] \circ \operatorname{Ad}\left[\alpha^{M-1}(g)\right] \circ \alpha^{M} .
\end{gathered}
$$

Hence, in view of

$$
\varphi^{\circ} \alpha^{M-1}=(\operatorname{Ad}[g] \circ \alpha)^{M-1} \circ \varphi=\operatorname{Ad}\left[g_{M-1}\right] \circ \alpha^{M-1} \circ \varphi
$$

we obtain

$$
\begin{aligned}
\varphi^{\circ} \varphi^{\prime \prime} & =\operatorname{Ad}\left[\varphi\left(h_{M-1}\right) g_{M-1}\right] \circ \operatorname{Ad}\left[\alpha^{M-1}(\varphi(h) g)\right] \circ \alpha^{M} \\
& =\operatorname{Ad}\left[\varphi\left(h_{M-1}\right) g_{M-1}\right] \circ \alpha^{M-1} \circ \operatorname{Ad}[\varphi(h) g] \circ \alpha
\end{aligned}
$$

and inducting upon $M$ 


$$
\varphi^{\circ} \varphi^{\prime \prime}=(\operatorname{Ad}[\varphi(h) g] \circ \alpha)^{M} .
$$

Equations (1), (2), (3), (4) indicate that $\varphi, \varphi^{\prime \prime}$ effect a shift equivalence of $\operatorname{Ad}[h] \circ \alpha$ to $\operatorname{Ad}[\varphi(h) g] \circ \alpha$ with lag $M$. Now it is easy to check (see also 1.3 in [11]) that $\varphi \circ \phi$ and $\alpha^{2 M N-M-N} \circ \psi^{\prime} \circ \varphi^{\prime \prime}$ effect a shift equivalence of $\alpha$ to $\operatorname{Ad}[\varphi(h) g] \circ \alpha$ with lag $2 M N$.

2.3. Proof of 1.3. If $(\varphi, m, g) \sim\left(\varphi^{\prime}, m^{\prime}, g^{\prime}\right)$ and $(\phi, n, h) \sim\left(\phi^{\prime}, n^{\prime}, h^{\prime}\right)$, then there exist $x, y \in G, S \geq m, m^{\prime}, T \geq n, n^{\prime}$ such that

$$
\begin{aligned}
x g & =g^{\prime} \alpha(x) \\
\varphi^{\prime} \circ \alpha^{S-m^{\prime}} & =\operatorname{Ad}[x] \circ \varphi^{\circ} \alpha^{S-m} \\
y h & =h^{\prime} \alpha(y) \\
\psi^{\prime} \circ \alpha^{T-n^{\prime}} & =\operatorname{Ad}[y] \circ \psi^{\circ} \alpha^{T-n} .
\end{aligned}
$$

Writing

$$
\begin{aligned}
\xi & =\varphi(h) g \\
\xi^{\prime} & =\varphi^{\prime}\left(h^{\prime}\right) g^{\prime} \\
\Delta & =S+T \\
M & =m+n \\
M^{\prime} & =m^{\prime}+n^{\prime}
\end{aligned}
$$

for short and bearing in mind that $\varphi, \varphi^{\prime}, \phi, \phi^{\prime}$ effect respective shift equivalences of $\alpha$ to $\operatorname{Ad}[g] \circ \alpha, \operatorname{Ad}\left[g^{\prime}\right] \circ \alpha, \operatorname{Ad}[h] \circ \alpha, \operatorname{Ad}\left[h^{\prime}\right] \circ \alpha$ respectively we observe that $\Delta \geq M, M^{\prime}$ and

$$
\begin{aligned}
& \varphi^{\prime} \circ \psi^{\prime} \circ \alpha^{\Delta-M^{\prime}}=\varphi^{\prime} \circ \psi^{\prime} \circ \alpha^{S-m^{\prime}} \circ \alpha^{T-n^{\prime}} \\
& =\varphi^{\prime} \circ \operatorname{Ad}\left[h^{\prime}{ }_{S-m^{\prime}}\right] \circ \alpha^{S-m^{\prime}} \circ \psi^{\prime} \circ \alpha^{T-n^{\prime}} \\
& =\operatorname{Ad}\left[\varphi^{\prime}\left(h_{S-m^{\prime}}^{\prime}\right)\right] \circ \varphi^{\prime} \circ \alpha^{S-m^{\prime}} \circ \psi^{\prime} \circ \alpha^{T-n^{\prime}} \\
& =\operatorname{Ad}\left[\varphi^{\prime}\left(h^{\prime}{ }_{S-m^{\prime}}\right)\right] \circ\left(\operatorname{Ad}[x] \circ \varphi^{\circ} \alpha^{S-m}\right) \circ\left(\operatorname{Ad}[y] \circ \psi^{\circ} \alpha^{T-n}\right) \\
& =\operatorname{Ad}\left[\varphi^{\prime}\left(h^{\prime}{ }_{S-m^{\prime}}\right) x \varphi\left(\alpha^{S-m}(y)\right)\right] \circ \varphi^{\circ} \alpha^{S-m} \circ \phi^{\circ} \alpha^{T-n} \\
& =\operatorname{Ad}\left[\varphi^{\prime}\left(h^{\prime}{ }_{S-m^{\prime}}\right) x \varphi\left(\alpha^{S-m}(y)\right)\right] \circ \varphi^{\circ} \operatorname{Ad}\left[h_{S-m}^{-1}\right] \circ \psi^{\circ} \alpha^{S-m} \circ \alpha^{T-n} \\
& =\operatorname{Ad}\left[\varphi^{\prime}\left(h_{S-m^{\prime}}^{\prime}\right) x \varphi\left(\alpha^{S-m}(y)\right) \varphi\left(h_{S-m}^{-1}\right)\right] \circ \varphi^{\circ} \psi^{\circ} \alpha^{\Delta-M} \text {. }
\end{aligned}
$$

Thus we have shown, that

$$
\left(\varphi^{\prime} \circ \phi^{\prime}\right) \circ \alpha^{\Delta-M^{\prime}}=\operatorname{Ad}[\mathscr{Z}] \circ\left(\varphi^{\circ} \phi\right) \circ \alpha^{\Delta-M}
$$


where

$$
\mathscr{Z}=\varphi^{\prime}\left(h_{S-m^{\prime}}^{\prime}\right) x \varphi\left(\alpha^{S-m}(y)\right) \varphi\left(h_{S-m}^{-1}\right) .
$$

Now we claim that

$(* *)$

$$
\mathscr{Z} \xi=\xi^{\prime} \alpha(\mathscr{Z})
$$

which shows, together with $(*)$, that

$$
(\varphi \circ \phi, m+n, \xi) \sim\left(\varphi^{\prime} \circ \phi^{\prime}, m^{\prime}+n^{\prime}, \xi^{\prime}\right)
$$

which was to be proven. To substantiate $(* *)$ we start with the right hand side thereof and demonstrate it to be equal to the left hand side:

$$
\begin{aligned}
& \xi^{\prime} \alpha(\mathscr{Z})=\varphi^{\prime}\left(h^{\prime}\right) g^{\prime} \alpha\left[\varphi^{\prime}\left(h_{S-m^{\prime}}^{\prime}\right) x \varphi\left(\alpha^{S-m}(y)\right) \varphi\left(h_{S-m}^{-1}\right)\right] \\
& =\varphi^{\prime}\left(h^{\prime}\right) g^{\prime} \alpha\left(\varphi^{\prime}\left(h_{s-m^{\prime}}^{\prime}\right)\right) \alpha(x) \alpha\left(\varphi\left(\alpha^{s-m}(y)\right)\right) \alpha\left(\varphi\left(h_{s-m}^{-1}\right)\right) \\
& =\varphi^{\prime}\left(h^{\prime}\right) \varphi^{\prime}\left(\alpha\left(h_{S-m^{\prime}}^{\prime}\right)\right) g^{\prime} \alpha(x) \alpha\left(\varphi\left(\alpha^{S-m}(y)\right)\right) \alpha\left(\varphi\left(h_{S-m}^{-1}\right)\right) \\
& =\varphi^{\prime}\left(h^{\prime} \alpha\left(h^{\prime}{ }_{s-m^{\prime}}\right)\right) x g \alpha\left(\varphi\left(\alpha^{s-m}(y)\right)\right) \alpha\left(\varphi\left(h_{s-m}^{-1}\right)\right) \\
& =\varphi^{\prime}\left(h^{\prime}{ }_{S-m^{\prime}} \alpha^{S-m^{\prime}}\left(h^{\prime}\right)\right) x g \alpha\left(\varphi\left(\alpha^{S-m}(y)\right)\right) \alpha\left(\varphi\left(h_{s-m}^{-1}\right)\right) \\
& =\varphi^{\prime}\left(h_{S-m^{\prime}}^{\prime}\right) \varphi^{\prime}\left(\alpha^{S-m^{\prime}}\left(h^{\prime}\right)\right) x g \alpha\left(\varphi\left(\alpha^{S-m}(y)\right)\right) \alpha\left(\varphi\left(h_{S-m}^{-1}\right)\right) \\
& =\varphi^{\prime}\left(h_{S-m^{\prime}}^{\prime}\right) x \varphi\left(\alpha^{S-m}\left(h^{\prime}\right)\right) g \alpha\left(\varphi\left(\alpha^{S-m}(y)\right)\right) \alpha\left(\varphi\left(h_{S-m}^{-1}\right)\right) \\
& =\varphi^{\prime}\left(h^{\prime}{ }_{S-m^{\prime}}\right) x \varphi\left(\alpha^{S-m}\left(h^{\prime}\right)\right) \varphi\left(\alpha^{S-m}(\alpha(y))\right) g \alpha\left(\varphi\left(h_{S-m}^{-1}\right)\right) \\
& =\varphi^{\prime}\left(h^{\prime}{ }_{S-m^{\prime}}\right) x \varphi\left(\alpha^{S-m}\left(h^{\prime} \alpha(y)\right)\right) \varphi\left(\alpha\left(h_{S-m}^{-1}\right)\right) g \\
& =\varphi^{\prime}\left(h_{S-m^{\prime}}^{\prime}\right) x \varphi\left(\alpha^{S-m}(y h)\right) \varphi\left(\alpha\left(h_{S-m}^{-1}\right)\right) g \\
& =\varphi^{\prime}\left(h^{\prime}{ }_{S-m^{\prime}}\right) x \varphi\left(\alpha^{S-m}(y)\right) \varphi\left(\alpha^{S-m}(h) \alpha\left(h_{S-m}^{-1}\right)\right) g \\
& =\varphi^{\prime}\left(h^{\prime}{ }_{S-m^{\prime}}\right) x \varphi\left(\alpha^{S-m}(y)\right) \varphi\left(h_{S-m}^{-1}\right) \varphi(h) g \\
& =\mathscr{L} \xi \text {. }
\end{aligned}
$$

In the following $\langle\varphi, m, g$, $\rangle$ will stand for the equivalence class in $\mathscr{G}^{\sim}(\alpha)$ containing the triple $(\varphi, m, g) \in \mathscr{G}^{\sim}(\alpha)$.

2.4. Proof of 1.4. It can be routinely checked that $\left\langle\operatorname{Id}_{G}, 0, e\right\rangle$ is a welldefined element of $\mathscr{G}(\alpha)$ and acts as a neutral element for the binary operation on $\mathscr{G}(\alpha)$. On the other hand, for any $\langle\varphi, m, g\rangle,\left\langle\varphi^{\prime}, m^{\prime}, g^{\prime}\right\rangle,\left\langle\varphi^{\prime \prime}, m^{\prime \prime}, g^{\prime \prime}\right\rangle$ $\in \mathscr{G}(\alpha)$ we have

$$
\begin{aligned}
& \left(\langle\varphi, m, g\rangle\left\langle\varphi^{\prime}, m^{\prime}, g^{\prime}\right\rangle\right)\left\langle\varphi^{\prime \prime}, m^{\prime \prime}, g^{\prime \prime}\right\rangle \\
& =\left\langle\varphi^{\circ} \varphi^{\prime}, m+m^{\prime}, \varphi\left(g^{\prime}\right) g\right\rangle\left\langle\varphi^{\prime \prime}, m^{\prime \prime}, g^{\prime \prime}\right\rangle
\end{aligned}
$$




$$
\begin{aligned}
& =\left\langle\varphi^{\circ} \varphi^{\prime} \circ \varphi^{\prime \prime}, m+m^{\prime}+m^{\prime \prime}, \varphi^{\circ} \varphi^{\prime}\left(g^{\prime \prime}\right) \varphi\left(g^{\prime}\right) g\right\rangle \\
& =\left\langle\varphi^{\circ}\left(\varphi^{\prime} \circ \varphi^{\prime \prime}\right), m+m^{\prime}+m^{\prime \prime}, \varphi\left(\varphi^{\prime}\left(g^{\prime \prime}\right) g^{\prime}\right) g\right\rangle \\
& =\langle\varphi, m, g\rangle\left\langle\varphi^{\prime} \circ \varphi^{\prime \prime}, m^{\prime}+m^{\prime \prime}, \varphi^{\prime}\left(g^{\prime \prime}\right) g^{\prime}\right\rangle \\
& =\langle\varphi, m, g\rangle\left(\left\langle\varphi^{\prime}, m^{\prime}, g^{\prime}\right\rangle\left\langle\varphi^{\prime \prime}, m^{\prime \prime}, g^{\prime \prime}\right\rangle\right) .
\end{aligned}
$$

Consequently the binary operation on $\mathscr{G}(\alpha)$ is associative. Next we demonstrate the existence of inverse elements: Given $\langle\varphi, m, g\rangle \in \mathscr{G}(\alpha)$ there exist $\varphi^{\prime}: G \rightarrow$ $G, M \in Z^{+}$such that

$$
\begin{aligned}
\varphi^{\circ} \alpha & =\operatorname{Ad}[g] \circ \alpha \circ \varphi \\
\alpha^{\circ} \varphi^{\prime} & =\varphi^{\prime} \circ \operatorname{Ad}[g] \circ \alpha \\
\varphi^{\prime} \circ & =\alpha^{M} \\
\varphi^{\circ} \varphi^{\prime} & =(\operatorname{Ad}[g] \circ \alpha)^{M} .
\end{aligned}
$$

We claim

$$
\langle\varphi, m, g\rangle^{-1}=\left\langle\alpha^{m} \circ \varphi^{\prime}, M, \alpha^{m} \varphi^{\prime}\left(g^{-1}\right)\right\rangle:
$$

Indeed

$$
\begin{aligned}
& \left\langle\alpha^{m} \circ \varphi^{\prime}, M, \alpha^{m} \varphi^{\prime}\left(g^{-1}\right)\right\rangle\langle\varphi, m, g\rangle \\
= & \left\langle\alpha^{m} \circ \varphi^{\prime} \circ \varphi, M+m, \alpha^{m} \varphi^{\prime}(g) \alpha^{m} \varphi^{\prime}\left(g^{-1}\right)\right\rangle \\
= & \left\langle\alpha^{m+M}, m+M, e\right\rangle \\
= & \left\langle\operatorname{Id}_{G}, 0, e\right\rangle
\end{aligned}
$$

and

$$
\begin{aligned}
& \langle\varphi, m, g\rangle\left\langle\alpha^{m} \circ \varphi^{\prime}, M, \alpha^{m} \varphi^{\prime}\left(g^{-1}\right)\right\rangle \\
= & \left\langle\varphi^{\circ} \alpha^{m} \circ \varphi^{\prime}, m+M, \varphi\left(\alpha^{m}\left(\varphi^{\prime}\left(g^{-1}\right)\right)\right) g\right\rangle \\
= & \left\langle\operatorname{Ad}\left[g_{m}\right] \circ \alpha^{m} \circ \varphi^{\circ} \varphi^{\prime}, m+M, \operatorname{Ad}\left[g_{m}\right] \circ \alpha^{m} \circ \varphi^{\circ} \varphi^{\prime}\left(g^{-1}\right) g\right\rangle \\
= & \left\langle\operatorname{Ad}\left[g_{m+M}\right] \circ \alpha^{m+M}, m+M, \operatorname{Ad}\left[g_{m+M}\right] \circ \alpha^{m+M}\left(g^{-1}\right) g\right\rangle \\
= & \left\langle\operatorname{Id}_{G}, 0, e\right\rangle
\end{aligned}
$$

(take $T=m+M, x=g_{m+M}^{-1}$, for instance).

\section{Proof of $\mathbf{1 . 5}$}

Let $X$ be a compact, smooth manifold, $a: X \rightarrow X$ be an expanding map with a fixed point $x_{*} \in X$. By an argument due to M. Shub and J. Tate (Theorem 4[I], [9]) 
it can be shown that for any $\mathrm{CW}$-complex $Y$ and any $b: Y \rightarrow Y$ with a fixed point $y_{*} \in Y$ and any $\theta: \pi_{1}\left(Y, y_{*}\right) \rightarrow \pi_{1}\left(X, x_{*}\right)$ with

$$
\theta \circ b_{\#}=a_{\#} \circ \theta
$$

there exists a unique map $f: Y \rightarrow X$ such that

$$
\begin{aligned}
f\left(y_{*}\right) & =x_{*} \\
f \circ b & =a \circ f
\end{aligned}
$$

and

$$
f_{\#}=\theta \text {. }
$$

The map uniquely determined in this manner by $\theta$ will be denoted $F(\theta)$. We note in particular that if $b=a:\left(X, x_{*}\right) \rightarrow\left(X, x_{*}\right)$ and $\theta=a_{*}: \pi_{1}\left(X, x_{*}\right) \rightarrow \pi_{1}\left(X, x_{*}\right)$ then

$$
F\left(a_{\#}^{n}\right)=a^{n}
$$

for any $n \in Z^{+}$. On the other hand for any fixed points $x_{1}, x_{2}, x_{3}$ of $a$ and group homomorphisms

$$
\theta: \pi_{1}\left(X, x_{2}\right) \rightarrow \pi_{1}\left(X, x_{1}\right)
$$

and

$$
\rho: \pi_{1}\left(X, x_{3}\right) \rightarrow \pi_{1}\left(X, x_{2}\right)
$$

subject to

$$
\begin{aligned}
& a_{\#}^{\circ} \theta=\theta^{\circ} a_{\#} \\
& a_{\#}^{\circ} \rho=\rho^{\circ} a_{\#}
\end{aligned}
$$

we obviously have

$$
F(\theta \circ \rho)=F(\theta) \circ F(\rho) .
$$

In the following, $a: X \rightarrow X$ will always stand for an expanding map of a compact smooth manifold and $x_{*} \in X$ for a fixed point of $a$. Let $p: \bar{X} \rightarrow X$ be the universal covering projection of $X$. We again choose an arbitrary $\overline{x_{*}} \in$ $p^{-1}\left(x_{*}\right)$ which we fix as of now.

By a method described in detail in [13], it is possible to index fixed points of $a$ by elements of $\pi_{1}\left(X, x_{*}\right)$ in a well-defined fashion: Consider for each $g=$ $\langle\lambda\rangle \in \pi_{1}\left(X, x_{*}\right)$ the unique covering transformation $i(\langle\lambda\rangle)$ of $p: \bar{X} \rightarrow X$ which 
sends $\overline{x_{*}}$ to $\bar{\lambda}(1)$ where $\bar{\lambda}$ is the unique path in $\bar{X}$ with $p \circ \bar{\lambda}=\lambda$ and $\bar{\lambda}(0)=\overline{x_{*}}$. It can be checked that $i$ is an isomorphism from $\pi_{1}\left(X, x_{*}\right)$ to the group of covering transformations of $p: \bar{X} \rightarrow X$. Since we can obtain a riemannian metric on $\bar{X}$ by lifting that on $X$ via $p$, we may assume that $p$ is a local isometry and covering transformations of $p$ are isometries. Now, if $\bar{a}$ is the unique lifting of $a$ under $p$ with $\bar{a}\left(\overline{x_{*}}\right)=\overline{x_{*}}$, the map $i(g) \circ \bar{a}$ is an expanding map and has a unique fixed point $\overline{x_{g}}$. We write $x_{g}=p\left(\overline{x_{g}}\right)$ and note that $x_{g}$ is a fixed point of $a$. In fact ([13]) every fixed point of $a$ is of the form $x_{g}$. In particular $x_{*}=x_{e}$. Furthermore $x_{g}=x_{g^{\prime}}$ iff $g$ and $g^{\prime}$ are $a_{\#}$-equivalent, that is iff there exists $n \in \pi_{1}\left(X, x_{*}\right)$ with $n g=g^{\prime} a_{\#}(n)$.

Let $\bar{\omega}$ be a path in $\bar{X}$ with $\bar{\omega}(0)=\bar{x}_{g}, \bar{\omega}(1)=\overline{x_{*}}, \omega=p \circ \bar{\omega}$. It can be checked that $\omega^{-1} * a(\omega) \sim \lambda$. The homotopy class of $\omega$ depends only on $\langle\lambda\rangle$. We define

$$
\delta_{g}: \pi_{1}\left(X, x_{*}\right) \rightarrow \pi_{1}\left(X, x_{g}\right)
$$

by

$$
\delta_{g}(\langle\xi\rangle)=\left\langle\omega * \xi * \omega^{-1}\right\rangle
$$

for any $\langle\xi\rangle \in \pi_{1}\left(X, x_{*}\right)$. The homomorphism $\delta_{g}$ makes the diagram

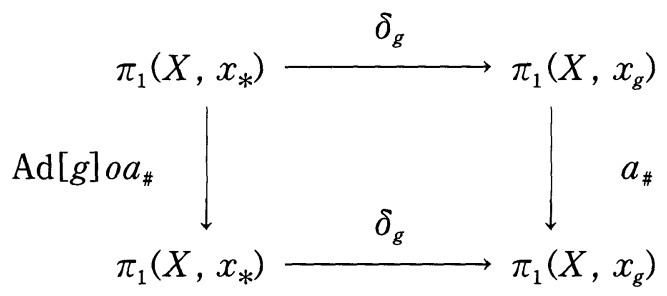

commutative. Moreover, if there exists $n \in \pi_{1}\left(X, x_{*}\right)$ such that $n g=$ $g^{\prime} a_{\#}(n)$ (equivalently $x_{g}=x_{g^{\prime}}$ ) then

$$
\delta_{g^{\prime}}^{-1} \circ \delta_{g}=\operatorname{Ad}[n]
$$

Let $Y, W$ be any spaces and $b: Y \rightarrow Y, c: W \rightarrow W$ any maps. Given any $F:$ $Y \rightarrow W$ with $F \circ b=c \circ F$ we define

$$
F_{\infty}: \lim _{\leftarrow}(Y, b) \rightarrow \underset{\leftarrow}{\lim }(W, c)
$$

by

$$
F_{\infty}\left(\left(y_{i}\right)_{i \in Z^{+}}\right)=\left(F\left(y_{i}\right)\right)_{i \in Z^{+}} .
$$

We note in particular that for $a^{k}: Y=X \rightarrow W=X$ we have $\left(a^{k}\right)_{\infty}=\sigma_{a}^{-k}$ for any 
$k \in Z^{+}$.

Now consider the map

$$
L: \mathscr{G}\left(\alpha_{\#}\right) \rightarrow \operatorname{Aut}\left(\sigma_{a}\right)
$$

defined by

$$
L(\langle\varphi, m, g\rangle)=\left(F\left(\delta_{g}{ }^{\circ} \varphi\right)\right)_{\infty}{ }^{\circ} \sigma_{a}^{m} .
$$

3.1. Lemma. $L$ is a well-defined and injective map.

Proof. First we observe that as $\varphi$ effects a shift equivalence of $a_{\#}: \pi(X$, $\left.x_{*}\right) \rightarrow \pi_{1}\left(X, x_{*}\right)$, to $\operatorname{Ad}[g] \circ a_{\#}: \pi_{1}\left(X, x_{*}\right) \rightarrow \pi_{1}\left(X, x_{*}\right)$, there exist $M \in Z^{+}$and $\varphi^{\prime}: \pi_{1}\left(X, x_{*}\right) \rightarrow \pi_{1}\left(X, x_{*}\right)$ such that $\varphi^{\prime} \circ \varphi=a_{\sharp}^{M}$. Consequently

$$
\begin{aligned}
\left(F\left(\varphi^{\prime} \circ \delta_{g}^{-1}\right)\right)_{\infty} \circ\left(F\left(\delta_{g} \circ \varphi\right)\right)_{\infty} & =\left(F\left(\varphi^{\prime} \circ \delta_{g}^{-1}\right) \circ F\left(\delta_{g} \circ \varphi\right)\right)_{\infty} \\
& =\left(F\left(\varphi^{\prime} \circ \delta_{g}^{-1} \circ \delta_{g} \circ \varphi\right)\right)_{\infty} \\
& =\left(F\left(a_{\#}^{M}\right)\right)_{\infty} \\
& =\left(\left(a^{M}\right)\right)_{\infty} \\
& =\sigma_{a}^{-M} .
\end{aligned}
$$

Therefore $\left(F\left(\delta_{g} \circ \varphi\right)\right)_{\infty}$ has a continuous inverse. Since $\sum$ is compact $\left(F\left(\delta_{g} \circ \varphi\right)\right)_{\infty}$ is a homeomorphism which commutes with $\sigma_{a}$ by its definition. Secondly, if $\langle\varphi, m$, $g\rangle=\left\langle\varphi^{\prime}, m^{\prime}, g^{\prime}\right\rangle$, then there exist $T \in Z$ with $T \geq m, m^{\prime}$ and $n \in \pi_{1}\left(X, x_{*}\right)$ such that

$$
\begin{aligned}
n g & =g^{\prime} a_{\#}(n) \\
\varphi^{\prime} \circ a_{\#}^{T-m^{\prime}} & =\operatorname{Ad}[n] \circ \varphi^{\circ} a_{\#}^{T-m} .
\end{aligned}
$$

In this case we have $x_{g}=x_{g^{\prime}}$ on the one hand and $\delta_{g^{\prime}}^{-1} \circ \delta_{g}=\operatorname{Ad}[n]$ on the other. Hence

$$
\delta_{g^{\prime}} \circ \varphi^{\prime} \circ a_{\#}^{T-m^{\prime}}=\delta_{g} \circ \varphi^{\circ} a_{\#}^{T-m}
$$

which gives

$$
\left(F\left(\delta_{g^{\circ}} \circ \varphi^{\prime}\right)\right)_{\infty}{ }^{\circ} \sigma_{a}^{-\left(T-m^{\prime}\right)}=\left(F\left(\delta_{g}^{\circ} \varphi\right)\right)_{\infty}^{\circ} \sigma_{a}^{-(T-m)}
$$

or

$$
\left(a^{T}\right)_{\infty} L\left(\left\langle\varphi^{\prime}, m^{\prime}, g^{\prime}\right\rangle\right)=\left(a^{T}\right)_{\infty}{ }^{\circ} L(\langle\varphi, m, g\rangle) .
$$

Therefore we conclude that 


$$
L\left(\left\langle\varphi^{\prime}, m^{\prime}, g^{\prime}\right\rangle\right)=L(\langle\varphi, m, g\rangle)
$$

since $\left(a^{T}\right)_{\infty}$ is a homeomorphism. The above proof of well-definedness, reading backwards, shows that $L$ is injective, too.

3.2. LEMMA.

$$
F\left(\delta_{g} \circ \varphi\right) \circ F\left(\delta_{h}^{\circ} \psi\right)=F\left(\delta_{\varphi(h) g}{ }^{\circ} \varphi^{\circ} \psi\right)
$$

Proof. Let's write $F=F\left(\delta_{g}{ }^{\circ} \varphi\right), G=F\left(\delta_{h}{ }^{\circ} \psi\right)$ for short. Let $\bar{F}: \bar{X} \rightarrow \bar{X}, \bar{G}:$ $\bar{X} \rightarrow \bar{X}$ be the respective liftings of $F, G$ with $\bar{F}\left(\overline{x_{*}}\right)=\bar{x}_{g}, \bar{G}\left(\overline{x_{*}}\right)=\overline{x_{h}}$. Consider paths $\bar{\tau}, \bar{\omega}$ in $\bar{X}$ with $\bar{\tau}(1)=\bar{\omega}(1)=\overline{x_{*}}, \bar{\tau}(0)=\overline{x_{h}}, \bar{\omega}(0)=\overline{x_{g}}$. Writing $\bar{\eta}=$ $\bar{F}(\bar{\tau}) * \bar{\omega}, \eta=p^{\circ} \bar{\eta}, \tau=p^{\circ} \bar{\tau}, \omega=p \circ \bar{\omega}$ we observe that

$$
\begin{gathered}
\left.\bar{\eta}(0)=\bar{F}(\bar{\tau}(0))=\bar{F}\left(\overline{x_{h}}\right)\right)=\bar{F} \circ \bar{G}\left(\overline{x_{*}}\right) \\
\bar{\eta}(1)=\bar{\omega}(1)=\overline{x_{*}} \\
\eta=F(\tau) * \omega
\end{gathered}
$$

and

$$
F \circ G\left(x_{*}\right)=x_{u}
$$

where

$$
\begin{aligned}
\langle u\rangle & =\left\langle\eta^{-1} * a(\eta)\right\rangle \\
& =\left\langle\omega^{-1} * F\left(\tau^{-1}\right) * a(F(\tau)) * a(\omega)\right\rangle \\
& =\left\langle\omega^{-1} * F\left(\tau^{-1} * a(\tau)\right) * \omega\right\rangle\left\langle\omega^{-1} * a(\omega)\right\rangle \\
& =\varphi(h) g
\end{aligned}
$$

in view of the fact that

$$
\varphi=\delta_{g}^{-1} \circ F_{\#}
$$

Hence

$$
\varphi(\langle\xi\rangle)=\left\langle\omega^{-1} * F(\xi) * \omega\right\rangle
$$

for any $\langle\xi\rangle \in \pi_{1}\left(X, x_{*}\right)$. Similarly we have

$$
\phi=\delta_{1}^{-1} \circ G_{\#}
$$

and

$$
\varphi(\langle\xi\rangle)=\left\langle\tau^{-1} * F(\xi) * \tau\right\rangle
$$


for any $\langle\xi\rangle \in \pi_{1}\left(X, x_{*}\right)$. Finally writing $H=F \circ G$, we note that

$$
\begin{aligned}
\delta_{\varphi(h) g}^{-1} \circ H_{\#}(\langle\xi\rangle) & =\delta_{\varphi(h) g}^{-1}{ }^{\circ} F_{\#} G_{\#}(\langle\xi\rangle) \\
& =\left\langle\eta^{-1} * F G(\xi) * \eta\right\rangle \\
& =\left\langle\omega^{-1} * F\left(\tau^{-1}\right) * F(G(\xi)) * F(\tau) * \omega\right\rangle \\
& =\varphi \circ \psi(\langle\xi\rangle) .
\end{aligned}
$$

By the uniqueness clause of Theorem 4.1 of [9] we conclude

$$
F\left(\delta_{\varphi(h) g}{ }^{\circ} \varphi^{\circ} \psi\right)=H_{\#}=F\left(\delta_{g} \circ \varphi\right) \circ\left(\delta_{h} \circ \psi\right) .
$$

3.3. LEMMA. $L: \mathscr{G}\left(\alpha_{\#}\right) \rightarrow \operatorname{Aut}\left(\sigma_{a}\right)$ is a group homomorphism.

Proof. This is now obvious by 3.2 .

The following lemma can be deduced from the lemmata 1, 4, 6 of [9] and the fact that $p: \bar{X} \rightarrow X$ is a local isometry. We include an elementary proof for the sake of completeness.

A continuous map $c: W \rightarrow W$ is said to be positively expansive if the space $W$ admits a metric $d$ and a number $\varepsilon>0$ such that $d\left(c^{n}(w), c^{n}\left(w^{\prime}\right)<\varepsilon\right.$ for all $n \in$ $Z^{+}$iff $w=w^{\prime}([7]$, [3]). If $W$ is a compact metric space, then this definition is independent of the choice of the metric.

\subsection{Lemma. Expanding maps of compact manifolds are positively expansive.}

Proof. Given an expanding map $a: X \rightarrow X$ of a compact manifold, we can choose a riemann metric and a constant $\lambda>1$ such that

$$
\|\operatorname{Ta}(x)(V)\|_{a(x)} \geq \lambda\|V\|_{x}
$$

for any $x \in X$ and any $V \in T_{x} X([6])$. As $a$ is a covering projection ([6]), there exists $\varepsilon>0$ such that $d\left(x, x^{\prime}\right)<\varepsilon$ and $a(x)=a\left(x^{\prime}\right)$ together imply $x=x^{\prime}$. We claim that the metric $d$ induced by the above suitably chosen riemann metric and $\varepsilon / 2>0$ do the trick: Indeed, if $d\left(a^{n}(x), a^{n}\left(x^{\prime}\right)\right)<\frac{\varepsilon}{2}$ for all $n \in Z^{+}$, then for each particular $n \in Z^{+}$there exists a piecewise differentiable path $\omega$ such that $\omega(0)=a^{n+1}(x), \omega(1)=a^{n+1}\left(x^{\prime}\right)$ and 


$$
\begin{aligned}
\operatorname{length}(\omega) & =\int_{0}^{1}\|\dot{\omega}(t)\|_{\omega(t)} d t \\
& <d\left(a^{n+1}(x), a^{n+1}\left(x^{\prime}\right)\right) \cdot \frac{\lambda+1}{2} \\
& <\frac{\varepsilon}{2} \frac{\lambda+1}{2} .
\end{aligned}
$$

Let $\bar{\omega}$ be the unique path with $a \bar{\omega}=\omega, \bar{\omega}(0)=a^{n}(x)$. Clearly $\bar{\omega}$ is piecewise differentiable.

Since

$$
a(\bar{\omega}(1))=\omega(1)=a^{n+1}\left(x^{\prime}\right)=a\left(a^{n}\left(x^{\prime}\right)\right)
$$

and

$$
\begin{aligned}
d\left(a^{n}\left(x^{\prime}\right), \bar{\omega}(1)\right) & \leq d\left(a^{n}\left(x^{\prime}\right), a^{n}(x)\right)+d\left(a^{n}(x), \bar{\omega}(1)\right) \\
& \leq \frac{\varepsilon}{2}+d(\bar{\omega}(0), \bar{\omega}(1) \\
& <\frac{\varepsilon}{2}+\operatorname{length}(\bar{\omega}) \\
& <\frac{\varepsilon}{2}+\frac{1}{\lambda} \operatorname{length}(\bar{\omega}) \\
& <\frac{\varepsilon}{2}+\frac{\lambda+1}{2 \lambda} \frac{\varepsilon}{2}<\varepsilon .
\end{aligned}
$$

We conclude that $\bar{\omega}(1)=a^{n}\left(x^{\prime}\right)$. Therefore

$$
\begin{aligned}
d\left(a^{n}(x), a^{n}\left(x^{\prime}\right)\right. & \leq \operatorname{length}(\bar{\omega}) \\
& <\frac{1}{\lambda} \operatorname{length}(\omega) \\
& <\frac{\lambda+1}{2 \lambda} d\left(a^{n+1}(x), a^{n+1}\left(x^{\prime}\right)\right) .
\end{aligned}
$$

This is true for each $n \in Z^{+}$and so

$$
d\left(x, x^{\prime}\right)<\left(\frac{\lambda+1}{2 \lambda}\right)^{n} d\left(a^{n}(x), a^{n}\left(x^{\prime}\right)\right) \leq\left(\frac{\lambda+1}{2 \lambda}\right)^{n} \frac{\varepsilon}{2} \rightarrow 0 .
$$

Consequently $d\left(x, x^{\prime}\right)=0$, equivalently $x=x^{\prime}$.

(It has been recently established, that essentially the converse of the above result is true, too: Every positively expansive map of a compact manifold is topolo- 
gically conjugate to an expanding map ([5]).

3.5. Lemma. Let $Y, W$ be compact spaces, $b: Y \rightarrow Y$ a surjective map, $c: W \rightarrow$ $W$ a positively expansive map. If a map

$$
F: \lim _{\leftarrow}(Y, b) \rightarrow \lim (W, c)
$$

commutes with the shift maps, that is

$$
F \circ \sigma_{b}=\sigma_{c}^{\circ} F
$$

then there exists $N \in Z^{+}$and $f: Y \rightarrow W$ such that

$$
f \circ b=c \circ f
$$

and

$$
F=f_{\infty}{ }^{\circ} \sigma_{b}^{N}=\sigma_{c}^{N} \circ f_{\infty}
$$

Proof. $d$ will denote the metrics on $Y, W$ without causing any confusion, $d^{\sim}$ is the related metric on the respective inverse limits. There exists $\varepsilon>0$ such that $d\left(c^{n}(w), c^{n}\left(w^{\prime}\right)\right)<\varepsilon$ for all $n \in Z^{+}$implies $w=w^{\prime}$. Let

$$
A=\operatorname{diam}(Y)=\max \left\{d\left(y, y^{\prime}\right) \mid y, y^{\prime} \in Y\right\} .
$$

There exists $\delta=\delta(\varepsilon)$ such that $d^{\sim}\left(\eta, \eta^{\prime}\right)<\delta$ implies $d\left(F(\eta), F\left(\eta^{\prime}\right)\right)<\varepsilon$ for all $\eta, \eta^{\prime} \in \lim _{\leftarrow}(Y, b)$. Choose $N \in Z^{+}$with $A / 2^{N}<\delta$. We claim, that if

$$
F\left(\left(y_{i}\right)_{i \in Z^{+}}\right)=\left(w_{i}\right)_{i \in Z^{+}}
$$

then $w_{0}$ depends only on $y_{N}$ : Indeed, if $\eta=\left(y_{i}\right)_{i \in Z^{+}}, \eta^{\prime}=\left(y_{i}^{\prime}\right)_{i \in Z^{+}}$and

$$
\begin{aligned}
& F(\eta)=\left(w_{i}\right)_{i \in Z^{+}} \\
& F\left(\eta^{\prime}\right)=\left(w_{\imath}^{\prime}\right)_{i \in Z^{+}}
\end{aligned}
$$

where $y_{N}=y_{N}^{\prime}$, then for every $s \in Z^{+}$we have

$$
\begin{gathered}
d\left(\sigma_{b}^{-s}(\eta), \sigma_{b}^{-s}\left(\eta^{\prime}\right)\right)=d^{\sim}\left(\sigma_{b}^{-s}\left(\left(y_{i}\right)_{i \in Z^{+}}\right), \sigma_{b}^{-s}\left(\left(y_{i}^{\prime}\right)_{i \in Z^{+}}\right)\right) \\
=d^{\sim}\left(\left(b^{s}\left(y_{i}\right)\right)_{i \in Z^{+}},\left(b^{s}\left(y_{\imath}^{\prime}\right)\right)_{i \in Z^{+}}\right) \\
=\sum_{k=N+s+1}^{\infty} \frac{1}{2^{k}} d\left(y_{k}, y_{k}^{\prime}\right) \leq \frac{A}{2^{N}}<\delta .
\end{gathered}
$$

Consequently

$$
d\left(c^{s}\left(w_{0}\right), c^{s}\left(w_{0}^{\prime}\right)\right) \leq d^{\sim}\left(\sigma_{c}^{-s} F(\eta), \sigma_{c}^{-s} F\left(\eta^{\prime}\right)\right)<\varepsilon
$$


for all $s \in Z^{+}$, hence $w_{0}=w_{0}^{\prime}$. In exactly the same way whenever $F\left(\left(y_{i}\right)_{i \in Z^{+}}\right)=$ $\left(w_{i}\right)_{i \in Z^{+}}, w_{\imath}$ depends only on $y_{i+N}$ for each $i \in Z^{+}$. Hence

$$
F \circ \sigma_{b}^{-N}\left(\left(y_{\imath}\right)_{i \in Z^{+}}\right)=\left(f_{i}\left(y_{i}\right)\right)_{i \in Z^{+}}
$$

where each $f_{i}: Y \rightarrow W$ is obviously continuous and satisfies

$$
f_{i} \circ b=c \circ f_{i+1} \text {. }
$$

Finally, as

$$
F \circ \sigma_{b}^{-N} \circ \sigma_{b}=\sigma_{c} \circ F \circ \sigma_{b}^{-N}
$$

we find

$$
f_{i}\left(y_{i+1}\right)=f_{i+1}\left(y_{i+1}\right)
$$

for any $\left(y_{i}\right)_{i \in Z^{+}} \in \lim _{\leftarrow}(Y, b)$. Since $b$ is surjective, we conclude that $f_{i}=f_{i+1}$ for each $i \in Z^{+}$and letting $f=f_{0}$ obtain $F=f_{\infty}{ }^{\circ} \sigma_{b}^{N}$.

3.6. Proof of 1.5. In view of 3.1 and 3.3 it remains to check that $L$ is surjective: By 3.5 , for every $F \in \operatorname{Aut}\left(\sigma_{a}\right)$ there exist $f, f^{\prime}: X \rightarrow X$ commuting with $a$ and $m, n \in Z^{+}$such that

$$
\begin{gathered}
F=f_{\infty}{ }^{\circ} \sigma^{m} \\
F^{-1}=f^{\prime}{ }_{\infty}^{\circ} \sigma^{n} .
\end{gathered}
$$

It can be checked that $f, f^{\prime}$ effect a shift equivalence of $a:\left(X, x_{*}\right) \rightarrow\left(X, x_{*}\right)$ to $a:\left(X, x_{g}\right) \rightarrow\left(X, x_{g}\right)$ for some $g \in \pi_{1}\left(X, x_{*}\right)$ with $f\left(x_{*}\right)=x_{g}, f^{\prime}\left(x_{g}\right)=x_{*}$. Similarly it is seen that

$$
\varphi=\delta_{g}^{-1} \circ f_{\#}: \pi_{1}\left(X, x_{*}\right) \rightarrow \pi_{1}\left(X, x_{*}\right)
$$

and

$$
\phi=f_{\#}^{\prime} \delta_{g}: \pi_{1}\left(X, x_{*}\right) \rightarrow \pi_{1}\left(X, x_{*}\right)
$$

effect a shift equivalence of

$$
a_{\#}: \pi_{1}\left(X, x_{*}\right) \rightarrow \pi_{1}\left(X, x_{*}\right)
$$

to

$$
\operatorname{Ad}[g] \circ a_{\#}: \pi_{1}\left(X, x_{*}\right) \rightarrow \pi_{1}\left(X, x_{*}\right)
$$

and

$$
F=\left(F\left(\delta_{g}{ }^{\circ} \varphi\right)\right)_{\infty}{ }^{\circ} \sigma_{a}^{m}=L(\langle\varphi, m, g\rangle)
$$




\section{Proofs of $1.6,1.7$}

1.6 is rather straightforward. Yet we prove it in detail as a foretaste of and in preparation for the proof of 1.7 .

\subsection{Lemma. $\quad(\varphi(L), m, u) \in \mathscr{G}^{\sim}(\varphi(p))$ iff $P R(L) \subseteq P R(p)$.}

Proof. In view of the facts that all $\varphi(x)$ commute and that $Z$ is a commutative group it can be seen that $(\varphi(L), m, u) \in \mathscr{G}^{\sim}(\varphi(p))$ iff there exists $L^{\prime} \in Z$ and $n \in Z^{+}$such that $\varphi(L) \varphi\left(L^{\prime}\right)=\varphi\left(L^{\prime}\right) \varphi(L)=\varphi\left(L L^{\prime}\right)=\varphi(p)^{n}=\varphi\left(p^{n}\right)$ or equivalently $L L^{\prime}=p^{n}$. Clearly this happens iff $P R(L) \subseteq P R(p)$.

4.2. Lemma. $(\varphi(L), m, u) \sim\left(\varphi\left(L^{\prime}\right), m^{\prime}, u^{\prime}\right)$ in $\mathscr{G}^{\sim}(\varphi(p))$ iff $L p^{-m}=L^{\prime} p^{-m^{\prime}}$ and $u-u^{\prime} \equiv 0(\bmod (p-1))$.

Proof. $(\varphi(L), m, u) \sim\left(\varphi\left(L^{\prime}\right), m^{\prime}, u^{\prime}\right)$ in $\mathscr{G}^{\sim}(\varphi(p))$ iff there exist $T \geq$ $m, m^{\prime}, x \in Z$ such that

$$
\begin{aligned}
x+u & =u^{\prime}+\varphi(p)(x) \\
\varphi\left(L^{\prime}\right) \circ \varphi(p)^{T-m^{\prime}} & =\operatorname{Ad}[x] \circ \varphi(L) \circ \varphi(p)^{T-m}
\end{aligned}
$$

or equivalently

$$
\begin{aligned}
u-u^{\prime} & =(p-1) x \\
L^{\prime} p^{T-m^{\prime}} & =L p^{T-m},
\end{aligned}
$$

which means that

$$
\begin{gathered}
u-u^{\prime} \equiv 0(\bmod (p-1)) \\
L^{\prime} p^{-m^{\prime}}=L p^{-m} .
\end{gathered}
$$

4.3. Proof of 1.6. By 1.5 we have

$$
\operatorname{Aut}\left(\sigma_{a(p)}\right) \simeq \mathscr{G}(\varphi(p)) .
$$

Let $p=(-1)^{\tau} p_{1}^{k_{1}} \cdots p_{s}^{k_{s}}$ be the unique factorisation of $p$ into its prime (positive!) factors $s=\# P R(p)$. Consider

$$
\mathscr{H}:\left(Z_{2} \oplus Z^{P R(p)}\right) \square_{\alpha(p)} Z_{p-1} \rightarrow \mathscr{G}(\varphi(p))
$$

defined by 


$$
\mathscr{H}\left(\rho,\left(\xi_{1}, \ldots, \xi_{s}\right), \zeta\right)=\langle\varphi(L), m, u\rangle
$$

where $L, m, u \in Z$ with

$$
\begin{gathered}
L p^{-m}=(-1)^{\rho} p_{1}^{\xi_{1}} \cdots p_{s}^{\xi_{1}} \\
u \equiv \zeta(\bmod (p-1)) .
\end{gathered}
$$

By 4.2 it is readily seen that this is a well-defined map which is obviously surjective in view of 4.1 . To see that $\mathscr{H}$ is a homomorphism, note that if

$$
\begin{aligned}
\mathscr{H}\left(\rho,\left(\xi_{1}, \ldots, \xi_{s}\right), \zeta\right) & =\langle\varphi(L), m, n\rangle \\
\mathscr{H}\left(\rho^{\prime},\left(\xi_{1}{ }^{\prime}, \ldots, \xi_{1}{ }^{\prime}\right), \zeta^{\prime}\right) & =\left\langle\varphi\left(L^{\prime}\right), m^{\prime}, n^{\prime}\right\rangle
\end{aligned}
$$

that is

$$
\begin{aligned}
L p^{-m} & =(-1)^{\rho} p_{1}^{\xi_{1}} \cdots p_{s}^{\xi_{s}} \\
L^{\prime} p^{-m^{\prime}} & =(-1)^{\rho^{\prime}} p_{1}^{\xi_{1}^{\prime}} \cdots p_{s}^{\xi_{s}^{\prime}}
\end{aligned}
$$

and

$$
\begin{gathered}
u \equiv \zeta(\bmod (p-1)) \\
u^{\prime} \equiv \zeta^{\prime}(\bmod (p-1))
\end{gathered}
$$

then

$$
L L^{\prime} p^{-\left(m+m^{\prime}\right)}=(-1)^{\rho+\rho^{\prime}} p_{1}^{\xi_{1}+\xi_{1}^{\prime}} \cdots p_{s}^{\xi_{s}+\xi_{s}^{\prime}}
$$

and

$$
\begin{gathered}
\varphi(L)\left(u^{\prime}\right)+u=u+L u^{\prime} \equiv \zeta+L \zeta^{\prime} \\
=\zeta+\alpha(p)\left(\rho,\left(\xi_{1} \cdots \xi_{s}\right)\right)\left(\zeta^{\prime}\right) .
\end{gathered}
$$

Consequently

$$
\begin{aligned}
& \mathscr{H}\left(\rho,\left(\xi_{1}, \ldots, \xi_{s}\right), \zeta\right) \mathscr{H}\left(\rho^{\prime},\left(\xi_{1}^{\prime}, \ldots, \xi_{s}^{\prime}\right), \zeta^{\prime}\right) \\
= & \langle\varphi(L), m, u\rangle\left\langle\varphi\left(L^{\prime}\right), m^{\prime}, u^{\prime}\right\rangle=\left\langle\varphi\left(L L^{\prime}\right), m+m^{\prime}, \varphi(L)\left(u^{\prime}\right)+u\right\rangle \\
= & \left\langle\varphi\left(L L^{\prime}\right), m+m^{\prime}, u+L u^{\prime}\right\rangle \\
= & \mathscr{H}\left(\rho+\rho^{\prime},\left(\xi_{1}+\xi_{1}^{\prime}, \ldots, \xi_{s}+\xi_{s}^{\prime}\right), \zeta+\alpha(p)\left(\rho,\left(\xi_{1}, \ldots, \xi_{1}\right)\right)\left(\zeta^{\prime}\right)\right) \\
= & \mathscr{H}\left(\left(\rho,\left(\xi_{1}, \ldots, \xi_{s}\right), \zeta\right)\left(\rho^{\prime},\left(\xi_{1}^{\prime}, \ldots, \xi_{s}^{\prime}\right), \zeta^{\prime}\right)\right) .
\end{aligned}
$$

Finally, ker $\mathscr{H}$ is trivial since

$$
\mathscr{H}\left(\rho,\left(\xi_{1}, \ldots, \xi_{s}\right), \zeta\right)=\langle\varphi(L), m, u\rangle=\langle\varphi(1), 0,0\rangle
$$

implies 


$$
(-1)^{\rho} p_{1}^{\xi_{1}} \cdots p_{s}^{\xi_{s}}=L p^{-m}=1
$$

equivalently

$$
\rho=0, \quad \xi_{1}=\xi_{1}=\cdots=\xi_{s}=0
$$

and

$$
\zeta=0 \quad(\bmod (p-1))
$$

Before going on to consider the proof of 1.7 , we observe

$$
\begin{gathered}
\varphi(p, q, r) \circ \varphi\left(p^{\prime}, q^{\prime}, r^{\prime}\right)=\varphi\left(p p^{\prime}, q+q^{\prime} p, r r^{\prime}\right) \\
\varphi(p, q, r)^{n}=\varphi\left(p^{n}, q\left(1+\cdots+p^{n-1}\right), r^{n}\right) \\
\operatorname{Ad}\left[A^{x} B^{y}\right] \circ \varphi(p, q, r)=\varphi\left((-1)^{y} p,(-1)^{y} q+2 x, r\right) .
\end{gathered}
$$

For further details, the reader is referred to ([11], [12]).

4.4 Lemma. $\left.\left(\varphi(L, M, N), m, A^{u} B^{v}\right) \in \mathscr{G}^{\sim}(\varphi(p, q, r))\right)$ iff

a) $P R(L) \subseteq P R(p), P R(N) \subseteq P R(r)$

b) $v=0(\bmod 2)$

c) $q(L-1)-2 u \equiv 0(\bmod (p-1))$

and

$$
M=\frac{q(L-1)-2 u}{p-1}
$$

Proof. $\quad\left(\varphi(L, M, N), m, A^{u} B^{v}\right) \in \mathscr{G}^{\sim}(\varphi(p, q, r))$ iff $\varphi(L, M, N)$ effects a shift equivalence of $\varphi(p, q, r)$ to $\operatorname{Ad}\left[A^{u} B^{v}\right] \circ \varphi(p, q, r)$, in other words, iff there exist $\varphi\left(L^{\prime}, M^{\prime}, N^{\prime}\right), S \in Z^{+}$, such that

$$
\begin{aligned}
& \varphi(L, M, N) \circ \varphi(p, q, r)=\operatorname{Ad}\left[A^{u} B^{v}\right] \circ \varphi(p, q, r) \circ \varphi(L, M, N) \\
& \text { (2) } \varphi\left(L^{\prime}, M^{\prime}, N^{\prime}\right) \circ \operatorname{Ad}\left[A^{u} B^{v}\right] \circ \varphi(p, q, r)=\varphi(p, q, r) \circ \varphi\left(L^{\prime}, M^{\prime}, N^{\prime}\right) \\
& \varphi\left(L^{\prime}, M^{\prime}, N^{\prime}\right) \circ \varphi(L, M, N)=(\varphi(p, q, r))^{S} \\
& \varphi(L, M, N) \circ \varphi\left(L^{\prime}, M^{\prime}, N^{\prime}\right)=\left(\operatorname{Ad}\left[A^{u} B^{v}\right] \circ \varphi(p, q, r)^{S}\right.
\end{aligned}
$$

which can be rewritten as

$$
\varphi(L p, M+g L, N r)=\varphi\left((-1)^{v} p L,(-1)^{v} q+2 u+(-1)^{v} M p, r N\right)
$$


whereby we immediately note and put into use

$$
v=0(\bmod 2)
$$

and

$$
\begin{aligned}
\varphi\left(L^{\prime} p, M^{\prime}+(q+2 u) L^{\prime}, N^{\prime} r\right) & =\varphi\left(p L^{\prime}, g+M^{\prime} p, r N^{\prime}\right) \\
\varphi\left(L L^{\prime}, M^{\prime}+M L^{\prime}, N^{\prime} N\right) & =\varphi\left(p^{s}, q\left(1+p+\cdots+p^{s-1}\right), r^{s}\right) . \\
\varphi\left(L L^{\prime}, M+M^{\prime} L, N N^{\prime}\right) & =\varphi\left(p^{s},(q+2 u)\left(1+p+\cdots+p^{s-1}\right), r^{s}\right) .
\end{aligned}
$$

Upon comparison of respective components and casting away the more obviously uninformative pieces we see that $\varphi(L, M, N)$ effects a shift equivalence of $\varphi(p, q, r)$ to $\operatorname{Ad}\left[A^{u} B^{v}\right] \circ \varphi(p, q, r)$ iff there exist $L^{\prime}, M^{\prime}, N^{\prime} \in Z, S \in Z^{+}$such that

$$
\begin{aligned}
M(p-1) & =q(L-1)-2 u \\
M^{\prime}(p-1) & =q\left(L^{\prime}-1\right)+2 u L^{\prime} \\
L^{\prime} L & =p^{s} \\
N^{\prime} N & =r^{s} \\
M^{\prime}+M L^{\prime} & =q\left(1+p+\cdots+p^{s-1}\right) \\
M+M^{\prime} L & =(q+2 u)\left(1+p+\cdots+p^{s-1}\right) .
\end{aligned}
$$

It can now be checked that $\left(1^{\prime \prime}\right)$ and $\left(2^{\prime \prime}\right)$ are equivalent in view of $\left(3^{\prime \prime}\right)$ and $\left(5^{\prime \prime}\right)$, $\left(6^{\prime \prime}\right)$ are redundant.

4.4 shows that $M$ is of an inessential character in $\left\langle\varphi(L, M, N), m, A^{u} B^{v}\right\rangle$. Indeed, if $L, u$ are given, $M$ is completely determined.

4.5. Lemma. $\quad\left(\varphi(L, M, N), m, A^{u} B^{v}\right) \sim\left(\varphi\left(L^{\prime}, M^{\prime}, N^{\prime}\right), m^{\prime}, A^{u^{\prime}} B^{v^{\prime}}\right)$ in $\mathscr{G}^{\sim}(\varphi(p, q, r))$ iff there exists $y \in Z$ such that

a) $v-v^{\prime}=y(r-1)$

b) $(-1)^{y} u-u^{\prime}-q \pi(y)=0(\bmod (p-1))$

c) $(-1)^{y} L p^{-m}=L^{\prime} p^{-m^{\prime}}$

d) $N r^{-m}=N^{\prime} r^{-m^{\prime}}$

where $\pi$ denotes the unique parity preserving map from $Z$ into $\{0,1\}$. 
Proof. $\quad\left(\varphi(L, M, N), m, A^{u} B^{v}\right) \sim\left(\varphi\left(L^{\prime}, M^{\prime}, N^{\prime}\right), m^{\prime}, A^{u^{\prime}} B^{v^{\prime}}\right)$ in $\mathscr{G}^{\sim}(\varphi(p, q, r))$ iff there exist $T, x, y \in Z$ such that

$$
T \geq m, m^{\prime}
$$

and

$$
A^{x} B^{y} A^{u} B^{v}=A^{u^{\prime}} B^{v^{\prime}} \varphi(p, q, r)\left(A^{x} B^{y}\right)
$$

(2) $\operatorname{Ad}\left[A^{x} B^{y}\right] \circ \varphi(L, M, N) \circ(\varphi(p, q, r))^{T-m}=\varphi\left(L^{\prime}, M^{\prime}, N^{\prime}\right) \circ(\varphi(p, q, r))^{T-m^{\prime}}$

Remembering that $v=0(\bmod 2)$ it is seen that $(1)$ is equivalent to

$$
\begin{aligned}
x+(-1)^{y} u & =u^{\prime}+p x \\
y+v & =v^{\prime}+r y .
\end{aligned}
$$

(2) is equivalent to

$$
\begin{aligned}
(-1)^{y} L p^{T-m} & =L^{\prime} p^{T-m^{\prime}} \\
M^{\prime}+q\left(1+p+\cdots+p^{T-m^{\prime}-1}\right) L^{\prime} & =(-1)^{y}\left[M-q\left(1+p+\cdots+p^{T-m-1}\right) L\right]+2 x \\
N^{\prime} r^{T-m^{\prime}} & =N r^{T-m} .
\end{aligned}
$$

It remains to observe that $(2.2)$ is redundant in view of the equations

$$
\begin{aligned}
M(p-1) & =p(L-1)-2 u \\
M^{\prime}(p-1) & =p\left(L^{\prime}-1\right)-2 u
\end{aligned}
$$

combined with (1.1), remembering $2 \pi(y)=1-(-1)^{y}$.

4.5 indicates that $u$ is fairly inessential in $\left\langle\varphi(L, M, N), m, A^{u} B^{v}\right\rangle \in$ $\mathscr{G}^{\sim}(\varphi(p, q, r))$, too. Indeed if $p \equiv 0(\bmod 2)$, then $\left\langle\varphi(L, M, N), m, A^{u} B^{v}\right\rangle$ is completely determined by $L, N, m, v$.

4.6. LEMMA.

$$
\left\langle\varphi(L, M, N), m, A^{u} B^{v}\right\rangle=\left\langle\varphi(-L,-M, N), m, A^{-u-q} B^{v+r-1}\right\rangle .
$$

Proof. This is obvious by 4.5 .

4.7. Proof of 1.7. By 1.5 we have

$$
\operatorname{Aut}\left(\sigma_{a(p, q, r)}\right) \simeq \mathscr{G}(\varphi(p, q, r)) .
$$


Let $p=(-1)^{\tau} p_{1}^{i_{1}} \cdots p_{s}^{i_{s}}, r=(-1)^{\mu} r_{1}^{j_{1}} \cdots r_{t}^{j_{t}}$ be unique factorisations of $p, r$ into their prime (positive) factors, $s=\# P R(p) t=P R(r)$.

Case 1. $p=0(\bmod 2)$ : Consider

$$
\mathscr{H}: Z^{P R(p)} \oplus\left[\left(Z_{2} \oplus Z^{P R(r)}\right) \complement_{\alpha(r)} Z_{r-1}\right] \rightarrow \mathscr{G}(\varphi(p, q, r))
$$

defined by

$$
\mathscr{H}\left(\left(\xi_{1} \cdots \xi_{s}\right),\left(\rho,\left(\eta_{1} \cdots \eta_{t}\right), \zeta\right)\right)=\left\langle\varphi(L, M, N), m, A^{u} B^{v}\right\rangle
$$

where

$$
\begin{aligned}
L p^{-m} & =p_{1}^{\xi_{1}} \cdots r^{\xi_{s}} \\
N r^{-m} & =(-1)^{\rho} r_{1}^{\eta_{1}} \cdots r_{t}^{\eta_{t}} \\
v & \equiv 0(\bmod 2) \text { and } j\left(\frac{v}{2}\right)=\zeta
\end{aligned}
$$

where $j$ is the unique ring-with-multiplicative-identity homomorphism from $Z$ to $Z_{r-1}$ and

$$
\begin{aligned}
u & =\frac{1}{2} p q(L-1)(\bmod (p-1)) \\
M & =\frac{q(L-1)-2 u}{p-1} .
\end{aligned}
$$

First we observe that $\mathscr{H}$ is well-defined; if $L^{\prime}, M^{\prime}, N^{\prime}, u^{\prime}, v^{\prime}, m^{\prime}$ are integers satisfying (1), (2), (3), (4), (5) then

$$
\begin{gathered}
L p^{-m}=L^{\prime} p^{-m^{\prime}} \\
N r^{-m}=N^{\prime} r^{-m^{\prime}} \\
\frac{v}{2}-\frac{v^{\prime}}{2} \equiv 0(\bmod (r-1)) \\
u-u^{\prime}=\frac{1}{2} p q\left[(L-1)-\left(L^{\prime}-1\right)\right] \\
=\frac{1}{2} p q\left(L-L^{\prime}\right) \\
=\frac{1}{2} p q\left(L p^{m}-L^{\prime} p^{m^{\prime}}\right) \\
=\frac{1}{2} p q\left[\left(L+L^{\prime}\right)\left(p^{m}-p^{m^{\prime}}\right)\right. \\
=0(\bmod (p-1))
\end{gathered}
$$


and the equations of 4.5 are fulfilled with $y=0(\bmod 2)$ and

$$
\left\langle\varphi(L, M, N), m, A^{u} B^{v}\right\rangle=\left\langle\varphi\left(L^{\prime}, M^{\prime}, N^{\prime}\right), m^{\prime}, A^{u^{\prime}} B^{v^{\prime}}\right\rangle .
$$

Secondly we check that $\mathscr{H}$ is surjective: Indeed, given any

$$
\left\langle\varphi(L, M, N), m, A^{u} B^{v}\right\rangle \in \mathscr{G}(\varphi(p, q, r))
$$

we have $P R(L) \subseteq P R(p), P R(N) \subseteq P R(r), v \equiv 0(\bmod 2)$. By 4.6 we may assume that $L$ is of the same sign as $p^{m}$ hence there exist, $\xi_{i}, \eta_{\imath} \in Z, \zeta \in Z_{r-1}, \rho$ $\in Z_{2}$ such that

$$
\begin{gathered}
L p^{-m}=p_{1}^{\xi_{1}} \cdots p_{s}^{\xi_{s}} \\
N r^{-m}=(-1)^{\rho} r_{1}^{\eta_{1}} \cdots r^{\eta_{t}} \\
v\left(\frac{v}{2}\right)=\zeta .
\end{gathered}
$$

Thirdly we check that $\mathscr{H}$ is a homomorphism: If

$$
\begin{aligned}
\mathscr{H}\left(\left(\xi_{1} \cdots \xi_{s}\right), \rho,\left(\eta_{1} \cdots \eta_{t}\right), \zeta\right) & =\left\langle\varphi(L, M, N), m, A^{u} B^{v}\right\rangle \\
\mathscr{H}\left(\left(\xi_{1}^{\prime} \cdots \xi_{s}{ }^{\prime}\right), \rho^{\prime},\left(\eta_{1}^{\prime} \cdots \eta_{t}{ }^{\prime}\right), \zeta^{\prime}\right) & =\varphi\left(L^{\prime}, M^{\prime}, N^{\prime}, m^{\prime}, A^{u \prime} B^{v \prime}\right\rangle
\end{aligned}
$$

then

$$
\begin{gathered}
\left(L L^{\prime}\right) p^{-\left(m+m^{\prime}\right)}=L p^{-m} L^{\prime} p^{-m^{\prime}}=p_{1}^{\xi_{1}+\xi_{1}{ }^{\prime}} \cdots p_{s}^{\xi_{s}+\xi_{1}} \\
\left(N N^{\prime}\right) r^{-\left(m+m^{\prime}\right)}=N r^{-m} N^{\prime} r^{-m^{\prime}}=(-1)^{\rho+\rho^{\prime}} r_{1}^{\eta_{1}+\eta_{1}{ }^{\prime}} \cdots r^{\eta_{t}+\eta_{t^{\prime}}} \\
j\left(\frac{v}{2}+N \frac{v^{\prime}}{2}\right)=\zeta+N \zeta^{\prime}=\xi+\alpha(r)\left(\rho,\left(\eta_{1} \cdots \eta_{t}\right)\right)\left(\zeta^{\prime}\right) \\
u+L u^{\prime}=\frac{1}{2} p q\left[(L-1)+L\left(L^{\prime}-1\right)\right]=\frac{1}{2} p q\left(L L^{\prime}-1\right)(\bmod (p-1)) .
\end{gathered}
$$

Consequently

$$
\begin{aligned}
& \mathscr{H}\left(\left(\xi_{1} \cdots \xi_{s}\right), \rho,\left(\eta_{1} \cdots \eta_{t}\right), \zeta\right) \mathscr{H}\left(\left(\xi_{1}^{\prime} \cdots \xi_{s}^{\prime}\right), \rho^{\prime}\left(\eta_{1}^{\prime} \cdots \eta_{t}^{\prime}\right), \zeta^{\prime}\right) \\
& =\left\langle\varphi(L, M, N), m, A^{u} B^{v}\right\rangle\left\langle\varphi\left(L^{\prime}, M^{\prime}, N^{\prime}\right), m^{\prime}, A^{u^{\prime}} B^{v^{\prime}}\right\rangle \\
& =\left\langle\varphi\left(L L^{\prime}, M+M^{\prime} L, N N^{\prime}\right), m, m^{\prime}, \varphi(L, M, N)\left(A^{u^{\prime}} B^{v^{\prime}}\right) A^{u} B^{v}\right\rangle \\
& =\left\langle\varphi\left(L L^{\prime}, M+M^{\prime} L, N N^{\prime}\right), m, m^{\prime}, A^{u+L u^{\prime}} B^{v+N r^{\prime}}\right\rangle \\
& \mathscr{H}\left(\left(\xi_{1}+\xi_{1}^{\prime} \cdots \xi_{s}+\xi_{s}^{\prime}\right), \rho+\rho^{\prime},\left(\eta_{1}+\eta_{1}^{\prime} \cdots \eta_{t}+\eta_{t}^{\prime}\right),\right. \\
& \left.\quad \xi+\alpha(r)\left(\rho,\left(\eta_{1} \cdots \eta_{t}\right)\right)\left(\zeta^{\prime}\right)\right) \\
& \left.=\mathscr{H}\left[\left(\xi_{1} \cdots \xi_{t}\right), \xi\right]\left[\left(\xi_{1}^{\prime} \cdots \xi_{s}^{\prime}\right), \rho^{\prime},\left(\eta_{1}^{\prime} \cdots \eta_{1}^{\prime}\right), \xi^{\prime}\right]\right) .
\end{aligned}
$$

Finally we check that ker $\mathscr{H}$ is trivial: If 
$\mathscr{H}\left(\left(\xi_{1} \cdots \xi_{s}\right), \rho,\left(\eta_{1} \cdots \eta_{t}\right), \xi\right)=\left\langle\varphi(L, M, N), m, A^{u} B^{v}\right\rangle=\left\langle\varphi(1,0,0), 0, A^{0} B^{0}\right\rangle$

then

$$
\begin{gathered}
L p^{-m}=p_{1}^{\xi_{1}} \cdots p_{s}^{\xi_{s}} \\
N r^{-m}=(-1)^{\rho} r_{1}^{\eta_{1}} \cdots r^{\eta_{t}} \\
j\left(\frac{v}{2}\right)=\zeta
\end{gathered}
$$

on the one hand and there exists $y \in Z$ with

$$
\begin{gathered}
(-1)^{y} L p^{-m}=1=(-1)^{y} p_{1}^{\xi_{1}} \cdots p_{s}^{\xi_{s}} \\
N r^{-m}=(-1)^{\rho} r_{1}^{\eta_{1}} \cdots r_{t}^{\eta_{t}} \\
v-0=y(r-1)
\end{gathered}
$$

on the other. We note that $y=0(\bmod 2), \xi_{1}=\cdots=\xi_{s}=0, \rho=0, \eta_{1}=\cdots$ $\eta_{t}=0$ and $\zeta=0$, which implies that ker $\mathscr{H}$ is trivial.

Case 2. $p=1(\bmod 2)$ In this case we are to consider

$$
\mathscr{H}:\left(Z_{2} \oplus Z^{P R(p)}\right) \oplus\left[\left(Z_{2} \oplus Z^{P R(r)}\right)\left[_{\alpha(r)} Z_{r-1}\right] \rightarrow \mathscr{G}(\varphi(p, q, r))\right.
$$

defined by

$$
\mathscr{H}\left(\theta,\left(\xi_{1} \cdots \xi_{s}\right), \rho,\left(\eta_{1} \cdots \eta_{t}\right), \zeta\right)=\left\langle\varphi(L, M, N), m, A^{u} B^{v}\right\rangle
$$

where

$$
\begin{gathered}
L p^{-m}=p_{1}^{\xi_{1}} \cdots p_{s}^{\xi_{s}} \\
N r^{-m}=(-1)^{\rho} r_{1}^{\eta_{1}} \cdots r_{t}^{\eta_{t}} \\
v=0(\bmod 2), \\
j\left(\frac{v}{2}\right)=\zeta \\
u=\frac{1}{2} p q(L-1)+\pi(\theta) \frac{p-1}{2}(\bmod (p-1)) .
\end{gathered}
$$

The proof is similar to that in Case 1 .

Some of the ideas of this work were conceived during a stay at the "Sonderforschungsbereich Stochastische Mathematische Modelle" of the University of Heidelberg under the auspices of the Deutsche Forschungsgemeinschaft. It is a pleasure to express my thanks to S. Irk and C. Koç for helping me with the computations of the last section. 


\section{REFERENCES}

[1] M. Boyle, D. Lind, D. Rudolph, The automorphism group of a shift of finite type, Trans. Amer. Math. Soc., 306 (1988), 71-114.

[2] M. Boyle, W. Krieger, Periodic points and automorphisms of the shift, Trans. Amer. Math. Soc., 302 (1987), 125-149.

[3] E. Coven, W. Reddy, Positively expansive maps of compact manifolds, Lecture Notes in Math., 819 (1980), 96-110.

[ 4 ] G. A. Hedlund, Endomorphisms and automorphisms of the shift dynamical system, Math. Systems Theory, 3 (1969), 320-375.

[5] K. Hiraide, Positively expansive maps and growth of fundamental groups, Proc. Amer. Math. Soc., 104 (1988), 934-941.

[6] K. Krżyzewski, W. Szlenk, On invariant measures for expanding differentiable mappings, Studia Math., 33 (1960), 83-92.

[7] W. L. Reddy, On positively expansive maps, Math. Systems Theory, 6 (1972), $76-81$.

[8] J. P. Ryan, The shift and commutativity, Math. Systems Theory, 6 (1972), 82-85.

[9] M. Shub, Endomorphisms of compact differentiable manifolds, Amer. J. Math., 91 (1969), 175-199.

[10] S. Smale, Differentiable dynamical systems, Bull. Amer. Math. Soc., 73 (1967), $747-817$.

[11] C. Tezer, The shift on the inverse limit of a covering projection, Israel J. Math., 59 (1987), 129-149.

[12] - Shape classification of Klein-bottle-like continua, Quart. J. Math. Oxford Ser. (2), 40 (1989), $225-243$.

[13] - On fixed points of dynamical systems, Proc. Amer. Math. Soc., 110 (1990), $263-268$.

[14] R. F. Williams, Classification of 1-dimensional attractors, Proc. Sympos. Pure Math., Amer. Math. Soc. Providence, R. I., 14 (1970), 341-361.

[15] — Expanding attractors, Inst. Hautes Etudes Sci. Publ. Math., 43 (1974), $169-203$. 